\section{A Pascal program to perform the Bonferroni multistage multiple-correlation procedure}

\author{
JOHN CROSBIE \\ Flinders University of South Australia \\ Bedford Park, South Australia
}

When researchers perform several bivariate correlations on a data set, they need to know which of these correlation coefficients are statistically significant. However, this assessment is not straightforward. The probability estimates that are output by computerized statistical packages and shown in statistical tables are calculated on the assumption that only one test has been performed, and the probability of making at least one Type 1 error increases with the number of tests performed (Larzelere \& Mulaik, 1977). This problem is analogous to that posed by performing multiple $t$ tests on a data set. Although several procedures are readily available (and routinely performed) to provide protection for multiple comparisons of means (see Keppel, 1982, chap. 8), no procedure is readily available to protect researchers from unacceptable levels of Type 1 error when multiple correlation tests are performed.

The present paper describes a computer program that performs such a protection procedure for multiple correlation tests (the Bonferroni multistage procedure), and thereby maintains the specified Type 1 error rate (alpha level) across a set (family) of correlations.

\section{Bonferroni Multistage Procedure}

Larzelere and Mulaik (1977) reviewed several protection procedures for multiple correlation tests and concluded that the Bonferroni multistage was the best for this purpose. The general Bonferroni method is based on the assumption that if there are $m$ correlations to be tested, then the probability level for acceptance of each coefficient should be the specified alpha level divided by $m$. For example, with five correlations to be tested with a familywise alpha level of .05 , only correlations with a probability of less than .01 would be accepted. If all correlation coefficients are nonsignificant, then this general Bonferroni procedure is appropriate; however, if some coefficients are significant (as would be usual), then this procedure is too conservative (Larzelere, 1975; cited in Larzelere \& Mulaik, 1977). To overcome this conservative bias, the general Bonferroni was modified, and the multistage Bonferroni resulted.

In the first stage of the multistage Bonferroni, the alpha level for acceptance of each of the $m$ tests is calcu-

The author's mailing address is: Psychology Discipline, School of Social Sciences, Flinders University of South Australia, Bedford Park, South Australia, Australia 5042. lated by dividing the specified familywise alpha level by $m$ (and then by 2 if two-tailed tests are desired), and the Stage $1\left(s_{1}\right)$ tests that are significant at this test alpha level are determined. At the next stage, a new test alpha level is calculated for acceptance of the $m$ minus $s_{1}$, remaining nonsignificant correlations. This process continues until no more tests are statistically significant at the test alpha level. Therefore, at each stage the specified familywise alpha level is divided by the number of nonsignificant correlations at that stage, until no additional correlations are significant.

Although the logic of the multistage Bonferroni is simple, its computation is not. With the algorithm presented by Larzelere and Mulaik (1977), the calculations are exceptionally tedious, prone to error, and somewhat inaccurate; interpolation of Gaussian distribution tables and rounding error limit the precision of the results. However, with the improved formulas suggested by Harris (1976), the computation is much more onerous and frustrating, and a computerized procedure becomes essential.

\section{A Pascal Program}

The program described here performs the multistage Bonferroni procedure simply and efficiently. Across a wide rage of sample sizes and alpha levels, Harris (1976) showed that the Peiser (1943) approximation of $t$ (used by Larzelere \& Mulaik, 1977) is less accurate than Zelen and Severo's (1964; cited in Harris, 1976) four-term approximation. Therefore, this four-term approximation has been implemented so that the present program will produce accurate results with both small and large samples and at all alpha levels.

Input. Before this progam can be used, the correlation coefficients to be tested must be stored in a data file with each coefficient separated by at least one blank space or carriage return (i.e., freefield). To allow the program to be run on most versions of Pascal (the departures from the standard are discussed below), all numbers that contain decimal points (e.g., correlation coefficients and alpha levels) must have a digit before the decimal point. For example, $.23,-.37$, and .05 must be entered as 0.23 , -0.37 , and 0.05 .

The program prompts for the name of the file that contains the coefficients, the name to be assigned to the output, and the title to be displayed on the output file. Next, the program prompts for the familywise alpha levels to be used, the number of cases $(N)$ for the family of correlations, and the number of correlations in this family. Finally, the program reads the coefficients and stores them in an array, performs the Bonferroni assessments, writes a report to the output file, and tells the user the name of the file that contains this report.

Output. A sample output is shown in the Appendix. The output is headed by the title provided by the user, the name of the file that contained the coefficients, and 
the correlation coefficients that were tested. A row of asterisks separates the output between alpha levels, and a row of hyphens separates the output within alpha levels. For each assessment, the program outputs: the familywise alpha level; the family $N$; the number of correlations to be tested at that stage; the resultant $z, t$, and critical correlation coefficient (critical $r$ ) for acceptance at the specified familywise alpha level; and the correlation coefficients that are greater than this critical $r$. Assessments are two-tailed, so that only the magnitude of the coefficient is important, not the direction. At each stage, the number of correlations to be tested is reduced by the number of correlations that were significant at the previous stage, a new critical $r$ is computed for this number of nonsignificant correlations, and the number of coefficients greater than this new critical $r$ is assessed, until a stage is reached at which no additional significant correlations emerge. For example, the Appendix shows for the .1 alpha level at Stage 1 , of the original 15 correlations, 4 were significant; therefore, at Stage 2, only 11 correlations were used to compute the new critical $r$. At this stage, 6 correlations were significant, so at Stage 3, only 9 correlations were tested. At Stage 4, no additional coefficients were significant, so the analysis stopped for the .1 alpha level, and analyses commenced with the next alpha level (.05). The Appendix illustrates the difference between the general and the multistage Bonferroni procedures. With the general Bonferroni for the .1 alpha level, the critical $r$ is .292 and four coefficients are significant (shown in Stage 1 in the Appendix). However, for the multistage Bonferroni at the .1 alpha elvel, the critical $r$ is .270 and seven coefficients are significant (shown in Stage 4 in the Appendix). Therefore, if some coefficients are significant, the multistage is less conservative than the general Bonferroni procedure.

Program Language and Requirements. The program is written in Pascal, and the only departures from standard Pascal (Jensen \& Wirth, 1978) are the use of the underscore character (_) in long variable and procedure names and the use of variable names that are unique after 8 but less than 32 characters. The program was developed (and has been used exclusively) on a PRIME 9955, 8-MB, 32-bit machine, but any machine that can accommodate the 300 lines of program code and the array that contains the correlation coefficients should be able to run the program satisfactorily. Even 8-bit machines should be able to use this program to process several hundred correlation coefficients, because very little memory is used except for the array that holds the correlation coefficients, and the size of this array (and therefore the memory requirements) can be adjusted by changing the constant declaration MAXCORRS on Page 1 of the program.

Availability. A program listing may be obtained at no cost from the author.

\section{REFERENCES}

HaRris, R. J. (1976). Hand computation of Bonferroni critical values. Perceptual \& Motor Skills, 42, 515-521.

Jensen, K., \& WiRTh, N. (1978). Pascal: User manual and report. Berlin: Springer-Verlag.

KEPPEL, G. (1982). Design and analysis: A researcher's handbook (2nd ed.). Englewood Cliffs, NJ: Prentice-Hall.

LARZELERE, R. E. (1975). An empirical investigation of three procedures for multiple significance tests of intercorrelations. Unpublished master's thesis, Georgia Institute of Technology, Atlanta, GA.

LARZELERE, R. E., \& Mulaik, S. A. (1977). Single-sample tests for many correlations. Psychological Bulletin, 84, 557-569.

PeISER, A. M. (1943). Asymptotic formulas for the significance levels of certain distributions. Annals of Mathematical Statistics, 14, 56-62.

Zelen, M., \& Severo, N. C. (1964). Probability functions. In M. Abramowitz \& I. A. Stegun (Eds.), Handbook of Mathematical Functions. Washington, DC: National Bureau of Standards. (Applied Mathematics Series No. 55)

\section{APPENDIX}

Sample Output for an Analysis of the Larzelere and Mulaik (1977) Data with Alpha Levels of .1 and .05

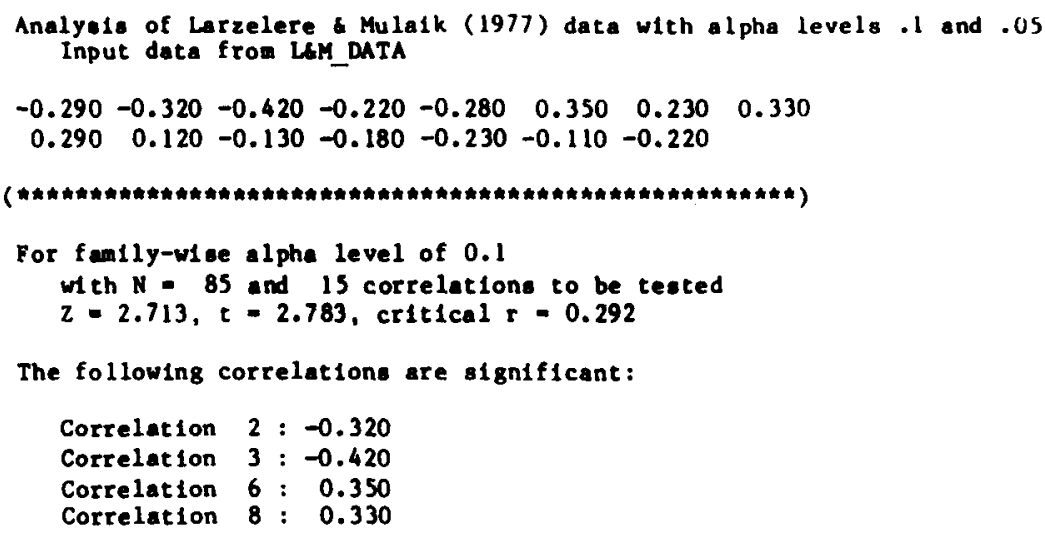


APPENDIX (continued)

For famlly-wise alpha level of 0.1

with $N=85$ and 11 correlations to be tested

$z=2.609, t=2.672$, critical $r=0.281$

The following correlations are significant:

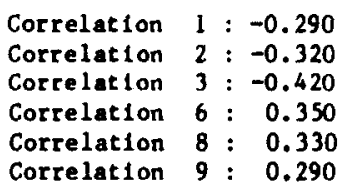

For fandly-wige alpha level of 0.1

wh th $N 85$ and 9 correlations to be tested

$z=2.540, t=2.598$, critical $r=0.274$

The following correlations are significant:

$\begin{array}{lll}\text { Correlation } & 1 & :-0.290 \\ \text { Correlation } & 2:-0.320 \\ \text { Correlation } & 3:-0.420 \\ \text { Correlation } & 5:-0.280 \\ \text { Correlattion } & 6: & 0.350 \\ \text { Correlation } 8: & 0.330 \\ \text { Correlation } & 9: & 0.290\end{array}$

For fandy-wise alpha level of 0.1

w1 th $N=85$ and 8 correlations to be tested

$z=2.498, t=2.554$, critical $r=0.270$

The following correlations are significant:
Correlation $1:-0.290$
Correlation $2:-0.320$
Correlation $3:-0.420$
Correlation $5:-0.280$
Correlation $6: 0.350$
Correlation $8: 0.330$
Correlation 9: 0.290

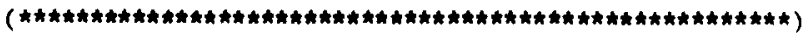

For family-wise alpha level of 0.05

with $N=85$ and 15 correlations to be tested

$z=2.936, t=3.023$, critical $r=0.315$

The following correlationg are significant:

$\begin{array}{llr}\text { Correlation } & 2:-0.320 \\ \text { Correlation } & 3: & -0.420 \\ \text { Correlation } & 6: & 0.350 \\ \text { Correlation } & 8: & 0.330\end{array}$

For faully-w1 se alpha level of 0.05

with $N=85$ and 11 correlations to be tested

$z=2.838, t=2.917, \mathrm{cr} t \mathrm{t}$ cal $r=0.305$

The following correlations are significant:

$\begin{array}{llr}\text { Correlation } & 2: & -0.320 \\ \text { Correlation } & 3: & -0.420 \\ \text { Correlation } & 6: & 0.350 \\ \text { Correlation } 8: & 0.330\end{array}$

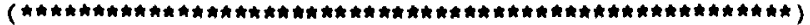

(Manuscript accepted for publication August 20, 1985.) 